\title{
The 3.5 ångström X-ray structure of the human connexin 26 gap junction channel is unlikely that of a fully open channel
}

\author{
Francesco Zonta ${ }^{1,2}$, Guido Polles ${ }^{3}$, Maria Federica Sanasi ${ }^{1}$, Mario Bortolozzi ${ }^{1,2}$ and Fabio Mammano ${ }^{1,2,4^{*}}$
}

\begin{abstract}
The permeability of gap junction channels to metabolites, and not simply to small inorganic ions, is likely to play an important role in development, physiology as well as in etiology of several diseases. Here, we combined dual patch clamp and fluorescence imaging techniques with molecular dynamics (MD) simulations to investigate the permeation of calcein, a relatively large fluorescent tracer (MW 622 Da) through homomeric gap junction channels formed by wild type human connexin26 ( $\mathrm{hCx} 26 \mathrm{wt}$ ) protomers. Our experimental data indicate that the unitary flux of calcein driven by a $125 \mu \mathrm{M}$ concentration difference is $J_{\text {pore }}=226$ molecule/s per channel. In the light of Eyring transition state theory adapted for the liquid phase, this value corresponds to an energy barrier of $\sim 20 k_{B} T$ (where $k_{B}$ is the Boltzmann constant and $T$ is absolute temperature). The barrier predicted by our MD simulations, based on the $3.5 \AA$ X-ray structural model of the hCx26wt gap junction channel, is $\sim 45 k_{B} T$. The main contributions to the energetics of calcein permeation originated from the interaction between the permeating molecule and the charged aminoacids lining the channel pore. Assigning a fake zero total charge to the calcein molecule yielded a value for the barrier height compatible with the experimental data. These results can be accounted for by two different (although not mutually exclusive) hypotheses: (1) the X-ray model of the hCx26wt gap junction channel is not representative of a fully open state; (2) post translational modifications affecting the $h C \times 26$ wt protein in our expression system differed from the modifications undergone by the proteins in the conditions used to obtain the crystal structure. Hypothesis (1) is compatible with data indicating that, only $10 \%$ or less of the channels forming a gap junction plaque are in the open state, and therefore the averaging procedure intrinsic in the generation of the crystal structure data more closely reflects that of a closed channel. Hypothesis (2) is compatible with recent mass spectrometry data and implies that the charge of several amino acid side chains may have been altered, thus modifying substantially the permeation properties of the channels in living cells.
\end{abstract}

Keywords: Connexin26, Calcein, Dual patch clamp, Fluorescence imaging, Umbrella sampling, Potential of mean force, Transition rate, Model

\section{Background}

Gap junction channels mediate communication between adjacent cells by allowing the passage of a variety of cytoplasmic molecules. They are formed by the head-to-head docking of two connexin protein hexamers, known as hemichannels or connexons, located in two adjacent cells [1]. Several studies showed that the permeation of

\footnotetext{
* Correspondence: fabio.mammano@unipd.it

'Department of Physics and Astronomy "G. Galilei", University of Padua, Padua 35131, Italy

${ }^{2}$ Venetian Institute of Molecular Medicine, Padua 35129, Italy

Full list of author information is available at the end of the article
}

cytoplasmic molecules through gap junction channels is fundamental in development and physiology, but also in the etiology of several diseases [2]. Second messengers, amino acids, nucleotides, glucose and its metabolites can permeate through at least some types of gap junction channels [3,4]. However, current understanding of the permeation properties and mechanisms is largely incomplete. Indeed, the unitary permeability of homomeric gap junction channels do not correlate well with ionic conductance and with presumptive pore sizes. The problem is exacerbated by the fact that gap junction channels in living cells can be formed by different connexin isoforms.

\section{Biomed Central}


Furthermore, most of the permeation properties of gap junction channels can, in principle, be dynamically regulated in response to external stimuli, such as voltage, $\mathrm{pH}$ or ionic concentrations [3,4].

The structure of connexin proteins and their assemblies was largely unknown until the publication of a model based on high resolution (3.5 ^) X-ray data of a hCx26wt channel [5]. The X-ray model permits to tackle issues left unresolved by previous models based on lower resolution data [6-9] such as the correct position of transmembrane helixes and the structure of extracellular regions. It also enables the study, by use of computational techniques, of ion permeation pathways $[10,11]$ and the prediction of unknown structures (wild type human connexin30, hCx30wt) [11].

With its 226 amino acids, hCx26wt is one of the smallest member of the connexin family. Mutations of GJB2, the gene encoding hCx26wt, are implicated in both syndromic and nonsyndromic deafness [12]. The X-ray data indicate that $\mathrm{hCx} 26 \mathrm{wt}$ comprises four transmembrane helixes (TM1, TM2, TM3 and TM4), which are connected by two extracellular loops (E1, E2) and one cytoplasm loop (CL) [5]. When assembled in hexamers, hCx26wt subunits create an aqueous pore in the plasma membrane, whose walls are formed by TM1 and TM2, plus the $\mathrm{N}$-terminus (NT) that folds inside the pore at the cytoplasmic mouth of the channel. The mouth is created by the CL and part of the NT and hosts several positively charged residues. On the extracellular side, instead, hCx26wt presents an accumulation of negatively charged residues [5].

Here, we measured the unitary permeability of homomeric gap junction channels formed by hCx26wt to calcein, a widely used inorganic fluorescent tracer. We paralleled the experimental work with MD simulations [11] based on the $3.5 \AA \mathrm{X}$-ray structure of hCx26wt [5]. Term of comparison between experiments and simulation is the transition rate of calcein through the channel, i.e. the number of calcein molecule that are able to traverse the channel per unit time. Our results indicate that the $3.5 \AA \mathrm{X}$-ray structure of hCx26wt is unlikely that of fully open channel and suggest that permeation properties of the channel may be significantly affected by post-translational modification of critical residues lining the pore.

\section{Results and discussion}

\section{Experimental determination of calcein transition rate}

We obtained an experimental estimate for the unitary flux of calcein using HeLa cells transiently transfected with cDNA encoding hCx26wt, as previously reported [13]. Calcein was delivered intracellularly, under whole-cell recording conditions, by passive diffusion out of a patch pipette filled with $125 \mu \mathrm{M}$ of this dye. Transfer of calcein between HeLa cell pairs coupled by homomeric hCx26wt channels was monitored by wide-field fluorescence microscopy followed by direct measurements of cell volume by digital optical sectioning [14]. Based on the measurement of the unitary permeability $p_{\mathrm{u}}=(3.0 \pm 1.0) \times 10^{-3}$ $\mu \mathrm{m}^{3} / \mathrm{s}$ (mean \pm standard error of the mean, SEM) presented in Figure 1, we estimate $J_{\text {pore }}=p_{\mathrm{u}}\left(c_{1}-c_{2}\right)=226 \pm$ 75 molecules/s per channel to be the unitary flux of calcein driven by a concentration difference $c_{1}-c_{2}=125 \mu \mathrm{M}$ (see Methods). A possible interpretation of this result is that, on average, a calcein molecule traverses a hCx26wt channel in a time $\tau_{\operatorname{Exp}}=1 / 226 \mathrm{~s}=4.4 \mathrm{~ms}$. We thus define the experimentally determined transition rate for calcein (with a concentration difference of $125 \mu \mathrm{M})$ as $k_{\operatorname{Exp}}=1 / \tau_{\operatorname{Exp}}=226 \mathrm{~s}^{-1}$.

\section{MD analysis of the permeation process}

Direct simulation of permeation of ions or molecules through a gap junction channel is beyond the current computational power due to the time scales involved (of the order of $\tau_{\text {Exp }}$, i.e. a few ms). For a system as large as

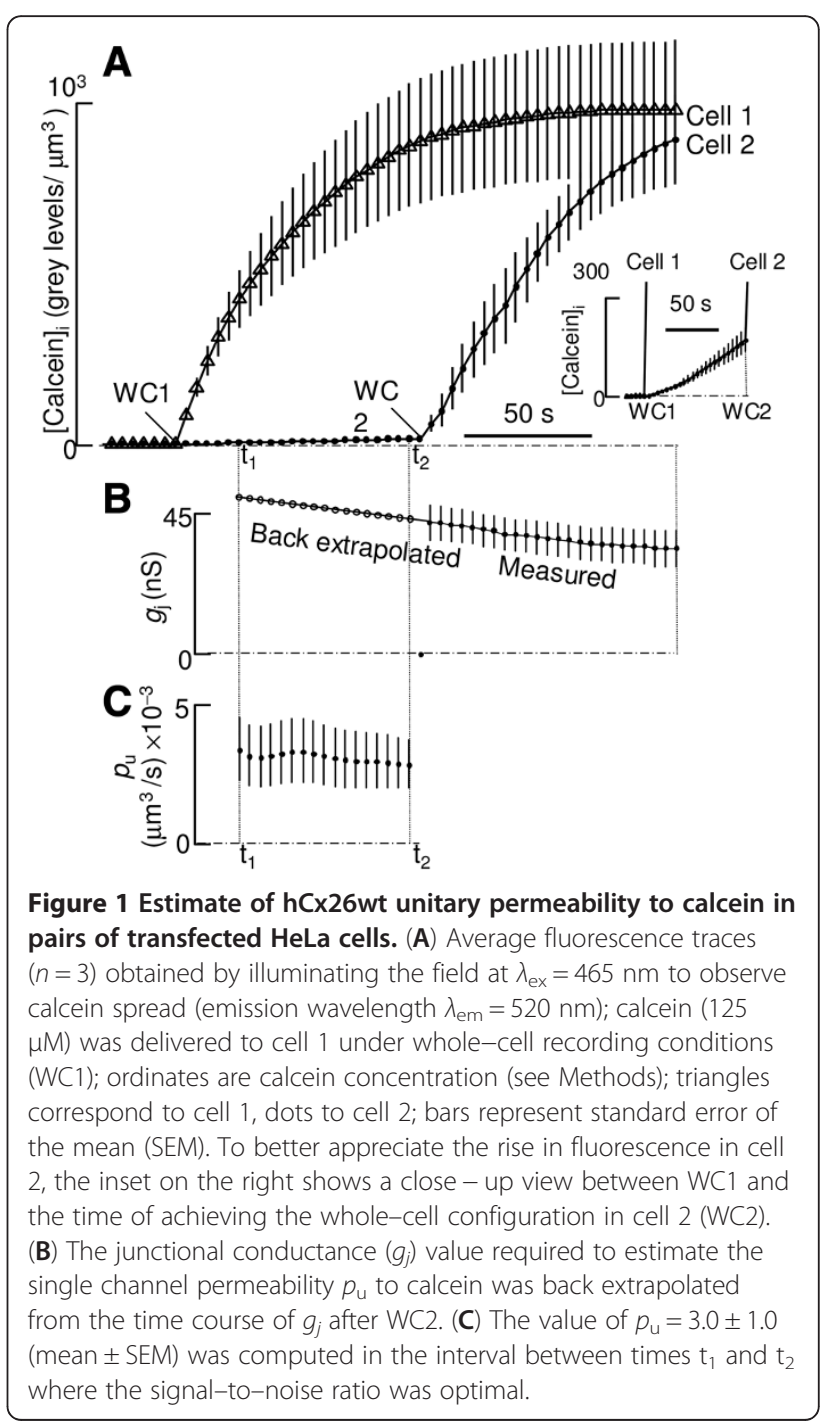


the one we are examining (with $>2 \times 10^{5}$ atoms) this exceeds by far the time window of state of the art MD simulations $(0.1$ to $1 \mu \mathrm{s})$. For this reason we used an indirect method based on the estimate of the free energy profile for the permeation process $[15,16]$, which can be approximated by the potential of mean force (PMF) [17] for calcein permeation through a homomeric hCx26wt hemichannel (Figure 2A, top panel). Initially, we computed this PMF using the umbrella sampling technique [18] (see Methods) assuming that all carboxyl groups of calcein are deprotonated, as expected at neutral $\mathrm{pH}$ in the bulk (i.e. when calcein is well solvated and essentially isolated within the solvent). At axial coordinate $z_{M}$, the PMF in Figure $2 \mathrm{~A}$ reaches a peak $W_{c}=45.2 k_{B} \mathrm{~T}$ (the subscript $c$ stands for "charged" calcein).

The calcein transition rate $k_{c}$, estimated from the PMF profile as described in the Methods, is twelve orders of



magnitude smaller than $k_{\text {Exp }}$, meaning that no calcein molecule would ever traverse a homomeric hCx26wt gap junction channel with the structure predicted by the $3.5 \AA \mathrm{X}$-ray data [5].

To find a rationale for the striking contrast between experimental results and MD model predictions we examined various possibilities. First, we noted that the channel pore narrows in the regions of fastest PMF increase (Figure 2A, middle panel), forcing calcein to interact with two positively charged residues, namely Lys41 and Met1 (Figure 2B). In our MD model, the latter has a protonated amino group because of its terminal position. Snapshots of calcein in four umbrella sampling windows spanning this critical region are presented in Figure 2 C. A particle moving in a channel with variable radius experiences a net entropic force due to the variation of the accessible phase space in the various segments of the channel. According to Zwanzig theory [19], this force is equivalent to a potential

$$
U_{S}(z)=-k_{B} T \ln \left(\frac{A(z)}{A\left(z_{0}\right)}\right)
$$

where $A$ is the section area of the channel, and $z_{0}$ a reference position. Function $U_{S}(z)$ for the hCx26wt connexon is plotted in Figure 2A (bottom panel). Not only is the maximum value of $U_{S}(\mathrm{z})$ significantly smaller than $W_{c}$, but $U_{S}(\mathrm{z})$ is also qualitatively different from the PMF profile. Based on this analysis we conclude that the entropic contribution to the PMF due to the variable radius of the pore is not the dominant factor and the channel is not closed from a purely entropic point of view.

Next we examined the issue of electrical charges. As noted above, in the course of our umbrella sampling simulations Met1 and Lys41 interacted with the negatively charged calcein molecule (Figure 2B). These interactions are further explored in Figure 3, showing that calcein carboxyl groups formed salt bridges with the amino groups of Met1 and Lys41. In the wider region of the narrowing pore, calcein formed bridges with Met1 alone (Figure 3A) or with both Met1 and Lys41 (Figure $3 \mathrm{~B}$ ); these bridges then remained stable for the rest of the dynamics. The region at TM1/E1 border barely accommodated one calcein molecule with a partial hydration shell. Here calcein interacted only with Lys41, which protruded into the pore (Figure 3C,D, left). However, due to the presence of six charged Lys41 side chains within this narrow region, the salt bridges were less stable, and tended to be exchanged between different protomers (Figure 3C,D, right). A calcein molecule needs to break and reform salt bridges to move within this region, and this explains the virtually impenetrable energy barrier that it encounters. 

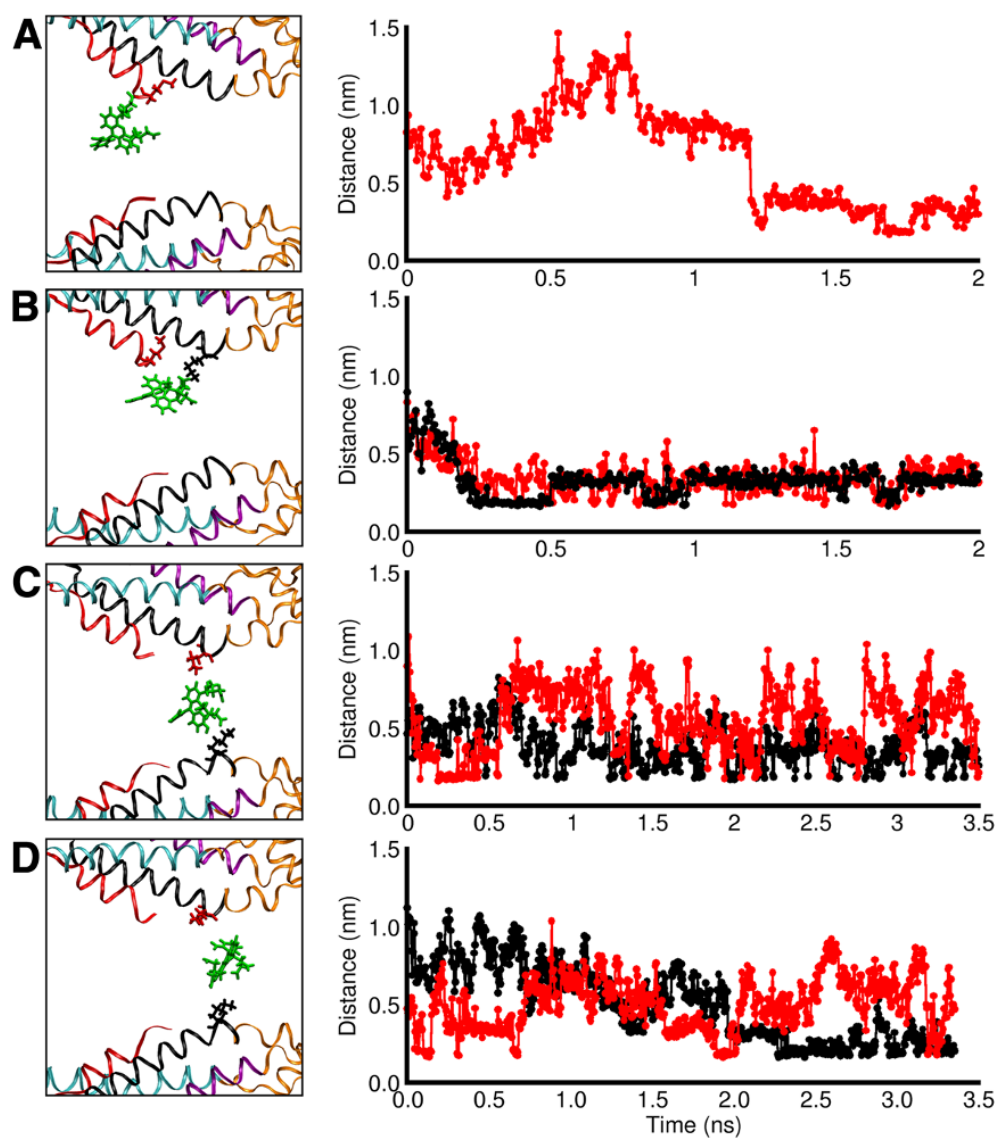

Figure 3 Formation of salt bridges in different umbrella sampling windows. Left panels (A-D) show side view representations of the calcein molecule in the channel pore; residues that interact with it are drawn in ball-and-stick style. Right panels (A-D) show time-course plots of the distance between one carboxy group of calcein and the amino group of interacting residues. (A) Calcein interacts with a Met1 alone. (B) Moving along the axis of the pore, calcein is found in a favorable position to interact both with Met1 and with Lys41. (C, D) After breaking the interaction with Met1, calcein interacts only with Lys41; in this zone, salt bridges are less stable due to calcein interacting simultaneously with Lys41 residues from different protomers.

However, there is evidence that several charged residues of hCx26wt can be modified by post translational modifications (Figure 4), which may also depend on cell condition such metabolic stress [10]. In particular it was shown that Met1 and seven lysines are acetylated (K15, K102, K103, K105, K108, K112 and K116), whereas three glutamic acids are gamma-carboxilated (E42, E47 and E114) [20]. Due to the difficulties of testing these sites, which requires building a new channel model for each candidate (and combinations thereof), we decided to reverse the point of view and cancel electrostatic interactions by eliminating calcein charges. We then performed a second set of simulations with calcein in a fully protonated state (i.e. with zero total charge). The results in Figure 5 show a PMF with a greatly reduced peak $W_{u}=19.6 k_{B} \mathrm{~T}$ (where the subscript $u$ stands for "uncharged" calcein), which corresponds to a transition rate $k_{u}=352 \mathrm{~s}^{-1}$ (see Methods), in far better agreement with $k_{\operatorname{Exp}}=226 \pm 75 \mathrm{~s}^{-1}$.

\section{Conclusions}

In this paper we measured the unitary flux of calcein through hCx26wt gap junction channels, and compared the experimentally determined value to that predicted by MD simulations based on the $3.5 \AA \mathrm{X}$-ray structural data [5]. Term of comparison is the unitary transition rate, i.e. the number of calcein molecules that are able to transit trough a single channel per unit time. Simulations were performed with two different charge states for the calcein molecule. In the first case calcein had all the carboxyl groups deprotonated, as expected at physiological $\mathrm{pH}$. In the other case, calcein was protonated and set to zero total charge. Our simulations indicate that a calcein molecule with a presumptive physiological charge is unable to traverse the channel due to the large energy barrier it faces $\left(45.2 k_{B} T\right)$. In contrast, the predicted transition rate for a calcein molecule with zero charge is compatible with the experimentally determined value. 


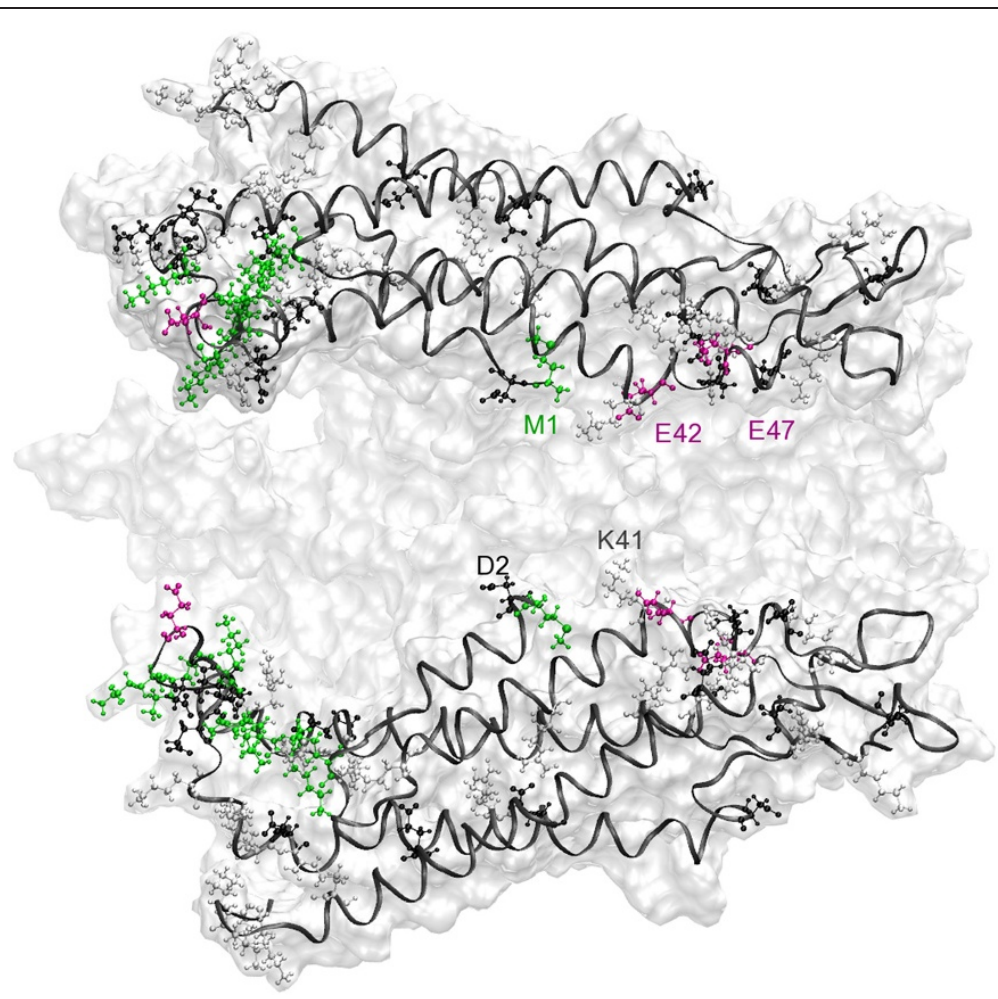

Figure 4 Post-translational modifications candidates. Amino acids which are candidates for post translational modifications, according to Ref. [20], and other charged amino acids are drawn in ball-and-stick style. Green: arginines and methionines that could be acetilated (global charge =0); magenta: glutamic acids that could be gamma-carboxilated (charge $=-2$ ); white: arginines and lysines (charge $=+1$ ); black: aspartic acids and glutamic acids (charge $=-1$ ).

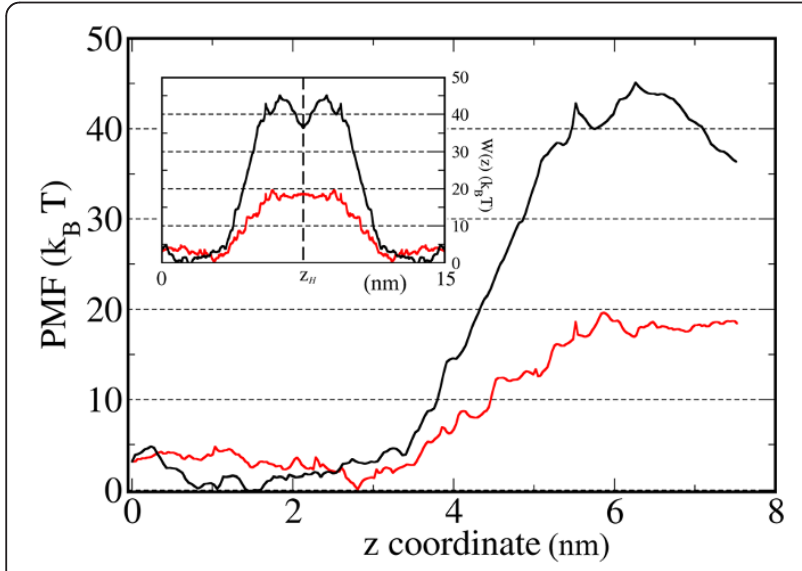

Figure 5 Comparison of PMFs for the permeation of charged (black) and uncharged calcein (red). The main graphs show PMF profiles for a hCx26wt hemichannel. Inset: corresponding free energy profiles, $W(z)$, for a complete gap junction channel obtained by reflecting the hemichannel PMFs about a vertical axis through the $z_{\mathrm{H}}$ abscissa.
Based on this analysis we conclude that the structural model of the hCx26wt channel derived from the $3.5 \AA \mathrm{X}$-ray data [5] is not permeable to calcein (even after MD relaxation) and the blockade is essentially electrostatic. Our conclusion is in contrast with the proposal of Maeda et al. [5] that the model represents an open channel. This proposal was based on the facts that: (i) unlike the M34A mutant channel structure [21], there are no obvious obstructions along the pore of the hCx26wt channel; (ii) the crystallization conditions adopted by Maeda et al. are compatible with the formation of channels in the open state (neutral $\mathrm{pH}$ without aminosulphonate buffer or any divalent ions). The discrepancy highlighted in the present work can be explained as follows: (1) there is no way to guarantee that the open channel structure was preserved during the partial dehydration and crystallization procedures; (2) in a gap junction plaque, only $10 \%$ or less of the channels are in an open state [22-24].

The following considerations lend further support to this conclusion. Structure relaxation during our MD simulations (carried out in a realistic environment) resulted in a widening of the pore, particularly at the cytoplasmic mouth of the channel [11]. Even this wider pore is impermeable to charged calcein. Moreover, as mentioned in the results, the charges of several residues facing the pore may be altered by post translation modifications, which may 
differ in the mammalian (HeLa) cells used in our experiments with calcein and in the insect cells used by Maeda et al. in their crystallization study [5]. A recent study showed that modification of the charge of these residues is sufficient to recover the correct current-voltage (I-V) relationship and ionic conductance, with the main role played by Met1 [10]. Among these, Met1, Glu42 and Glu47 are crucially located in the region of steepest PMF increase. Our results indicate that charged Met1 and Lys41 play a crucial role in hindering calcein permeation. Note that Lys41 is not a candidate for acetylation based on Ref. [20]. Furthermore, acetylation of this residue would reduce significantly the diameter of the pore at its narrowest point, thus we consider it unlikely. In this scenario, gammacarboxilation of Glu42 can be a fundamental determinant of channel permeability. Indeed, the carboxylated side chain of Glu42 is well poised for interacting with the amino group of Lys41. This interaction could stabilize the side chain of Lys41 (which instead appears rather mobile in our simulations) reducing the electrostatic potential felt by permeant molecules in the narrowest part of the channel, altogether favoring their transit. Further simulations are required to explore the influence of post translation modifications on the PMF of the channel not only for small inorganic ions but also for large permeant molecules.

\section{Methods}

\section{Experimental methods}

Dual whole cell patch-clamp recordings and fluorescence imaging

A detailed description of the theoretical and experimental framework for our permeability assays based on double patch-clamp and fluorescence imaging is provided in the Supplementary Methods of Ref. [13]. In brief, HeLa cells were co-transfected with a pcDNA3.1 expression vector carrying the coding region of $\mathrm{hCx} 26 \mathrm{wt}$ and an additional expression vector carrying a cytosolic CFP to identify transfected cells. An isolated pair of cells was contacted in the cell-attach configuration by patch-clamp pipettes loaded with calcein (Invitrogen, C481) at $125 \mu \mathrm{M}$ concentration. At time zero, the whole-cell configuration was established in cell 1 (WC1, Figure 1A), permitting the diffusion of calcein from cell 1 to cell 2 via overexpressed gap junction channels. The rise of fluorescence in cell 2, in addition to the estimates of the number $N_{\text {pore }}$ of open channels and the volume $V_{2}$ of cell 2, permitted us to derive the single channel permeability value $p_{\mathrm{u}}$ as

$$
p_{u}=\frac{V_{2}}{N_{\text {pore }}} \frac{d c / d t}{c_{1}-c_{2}}
$$

where $c_{1}$ is calcein concentration in cell 1 and $c_{2}<c_{1}$ is concentration in cell $2 . N_{\text {pore }}$ was derived by dividing the total junctional conductance $g_{\mathrm{j}}$ back extrapolated between
WC1 at WC2 (Figure 1B) by the previously determined single channel conductance $\gamma=115 \mathrm{pS}$ [13].

\section{Numerical simulation methods hCx26wt connexon MD model}

The fully atomistic model used for the hCx26wt hemichannel was developed in our previous work [11]. Briefly, we completed the published structure [5], adding the atoms that were missing in the original structure, and then inserting the initial hCx26 connexon configuration in a hole opened in a pre-relaxed membrane bilayer of phospholipids (palmytol posphatidyl choline, POPC). The final membrane configuration comprises 493 phospholipids. The positive net charge of the $\mathrm{hCx} 26$ connexon was neutralized with 54 chloride ions; additional pairs of potassium and chloride ions were added to mimic a physiological ionic strength. The system was solvated with a total of 39189 water molecules.

\section{Calcein parametrization}

The calcein molecule parameters required by our Molecular Dynamics simulations are not present in any standard library. We parameterized it as described below in two different protonation states: (i) standard charge, as reported by the manufacturer (Invitrogen, C481); (ii) completely protonated, i.e. zero total charge. The initial guess of calcein coordinates was obtained using the GlycoBioChem PRODRG2 server and the JME Molecular Editor provided on the server [25], from the molecular structure provided by the manufacturer of the calcein moiety used in the experiments (Invitrogen). After this step we refined the coordinates and obtained the parametrization for GAFF force field of the two different protonation state of calcein using the Antechamber package [26].

\section{Evalutation of PMFs by use of the umbrella sampling technique}

The PMF [17] is an approximation of the free energy changes along one or more reaction coordinates. One of the most frequently used and effective methods for computing it is the umbrella sampling technique $[18,27,28]$. The starting model used for the umbrella sampling simulation of calcein transition through the channel was taken from our previous work [11]. Initial configurations for each window of umbrella sampling were extracted from a steered Molecular Dynamics trajectory of the calcein transition through a hCx26wt hemichannel. In this preliminary simulation, the calcein molecule was dragged through the pore by an elastic force

$$
F(z, t)=-K_{\text {pull }}\left[z-\left(z_{0}+v t\right)\right]
$$

from the cytoplasmic to the extracellular side. Here $z$, our chosen reaction coordinate, is position along the pore axis, 
$K_{\text {pull }}=2000 \mathrm{~kJ} \mathrm{~mol}^{-1} \mathrm{~nm}^{-2}$ is the stiffness of a harmonic spring one end of which moved with constant velocity $v=10 \mathrm{~nm} / \mathrm{ns}$ (pull rate) along $z$ while the center of mass of the calcein molecule was attached to the opposite spring end and also restrained to move along the pore axis.

For the actual umbrella sampling simulations, window centers were initially spaced at $2 \AA$ and the whole simulated system underwent a short energy minimization process for each window. Thereafter we followed the MD trajectory, whereby the calcein center of mass was restrained by an elastic force

$$
F_{u m b, i}(z)=-K_{u m b}\left(z-z_{i}\right)
$$

where $K_{u m b}=1000 \mathrm{~kJ} \mathrm{~mol}^{-1} \mathrm{~nm}^{-2}$ is the elastic force constant and $z_{i}$ is the position of the $i$-th window center along $\mathrm{z}$ axis. The dynamics was initially followed for $1 \mathrm{~ns}$ for each window. After obtaining a preliminary PMF profile, we refined the spacing to $1 \AA$ and extended the duration of the simulated dynamics in the region were the PMFs in Figure 5 are rapidly increasing, until we reached convergence at 3.5 ns (meaning that the PMF profiles did not change appreciably by lengthening the simulation). The final total number of windows was $n=41$ for both charged and uncharged calcein. Overall, the simulation time used for evaluating the two PMF profiles was in excess of 200 ns. All MD simulations were performed with Gromacs 4.5 software [29] using the Amber03 force field, in the NTV ensemble [26]. Temperature was kept constant at $300 \mathrm{~K}$ using the Berendsen thermostat [30]. Particle Mesh Ewald summation [31] was used for the long-range electrostatic interactions, with a cut off of $1.0 \mathrm{~nm}$ for the direct interactions. The simulation time step was comprised between 1 and 2 fs.

\section{Estimate of transition rate from PMF}

We assimilated the permeation process through a complete gap junction channel to overcoming a free energy profile, $W(z)$, as described in Ref. [16]. We assumed $W(z)$ to be a symmetric function obtained by reflecting the hemichannel PMF in Figure 2A about a vertical axis through the $z_{H}$ abscissa corresponding to its extracellular end (see inset of Figure 5). Since both PMF peaks exceed by far the thermal energy (Figure 5), the transition rate can be derived by the liquid phase adaptation of the classical Eyring transition state theory $[15,16,32]$. We thus computed the transition rate, $k$, as

$$
k=\alpha k_{0} e^{-\left[W\left(z_{M}\right)-W\left(z_{0}\right)\right] / k_{B} T}
$$

where $z_{M}$ and $z_{0}$ are, respectively, the axial coordinate of the PMF maximum (in the extracellular vestibule) and minimum (in the cytoplasmic mouth of the channel; see Figure $2 \mathrm{~A}, \mathrm{~B})$. The prefactor $k_{0}$ is given by

$$
k_{0}=\sqrt{\frac{k_{B} T}{2 \pi m}}\left[\int_{z_{0}}^{z_{M}} e^{-W(z) / k_{B} T} d z\right]^{-1}
$$

where $m$ is the mass of the calcein molecule. A molecule that starts from $\mathrm{z}_{M}$ descends down the $W(z)$ profile pushed by a mean force

$$
F=-\frac{d W(z)}{d z}
$$

which favors its exit from the channel in either direction. This process is several orders of magnitude more probable than the reverse one, i.e. climbing $W(z)$ in the uphill direction. For this reason we computed the above integral over the interval $\left[\mathrm{z}_{0}, \mathrm{z}_{M}\right]$ considering that the rate-limiting step in the permeation process is determined by reaching coordinate $z_{M}$, i.e. the apex of the free energy barrier.

The product:

$$
k_{T S T} \equiv k_{0} e^{-\left[W\left(z_{M}\right)-W\left(z_{0}\right)\right] / k_{B} T}
$$

is the classical transition state theory rate in liquid phase $[15,16]$. It represents the frequency with which the calcein molecules reaches $z_{M}$ starting from $\mathrm{z}_{0}$.

A molecule that has reached $z_{M}$ moves forward, towards the opposite end of the complete channel, with a certain probability represented by the positive factor $\alpha<1$, also known as transmission coefficient. To estimate $\alpha$ we used a Brownian Dynamics approach in the presence of the external mean force $F$; namely, we numerically solved the motion equation:

$$
\Delta z=F \frac{D \Delta t}{k_{B} T}+\xi \sqrt{2 D \Delta t}
$$

where $\xi$ is a Gaussian white noise process, with zero mean and a time correlation function represented by Dirac's delta function [33]; $D=9.2 \times 10^{-7} \mathrm{~cm}^{2} / \mathrm{s}$, the diffusion coefficient of calcein, was taken from Ref. [34] as this figure is in good agreement with the value we obtained after a 100 ns MD simulation in the bulk. In this mean-fieldlike approximation, the channel was not allowed to fluctuate and it interacted with the calcein molecule only through $F$, while thermal fluctuations due to the collision with water molecules were synthetically taken into account in the diffusion coefficient and the random forces $\xi$. The time step for the Brownian Dynamics simulation was

Table 1 Summary of computed quantities

\begin{tabular}{llll}
\hline & $\boldsymbol{W}\left(\mathbf{z}_{\boldsymbol{M}}\right)\left(\boldsymbol{k}_{\boldsymbol{B}} \boldsymbol{T}\right)$ & $\boldsymbol{a}$ & $\boldsymbol{k}\left(\mathbf{s}^{\mathbf{- 1}}\right)$ \\
\hline Charged & 45.2 & 0.01 & $7.7 \times 10^{-11}$ \\
Uncharged & 19.6 & 0.16 & 352 \\
\hline
\end{tabular}


set to 1 ps. This coarse-grained model allowed us to simulate $10^{6}$ transitions of a calcein molecule with reasonable use of CPU time. We then estimated the transmission coefficient $\alpha$ as the fraction of simulations that yielded a calcein molecule on the opposite side of the complete gap junction channel. Table 1 summarize the computed quantities used in this work.

Final note: the estimates of the transition rates represent the number of transitions (per unit time) that occur under saturating conditions, i.e. when the wait time between successive transitions is null. To realize such conditions, the bulk calcein concentration in cell 1 must be such that (at least) one calcein molecule is present, at any given time, within the cytoplasmic vestibule of the channel. To assess whether saturation was achieved under our experimental conditions (with a calcein concentration in the patch pipette equal to $125 \mu \mathrm{m}$ ), we simulated the diffusion of calcein inside the cell as a Brownian random walk. The results of this independent set of simulations indicate that the number of calcein molecules diffusing from bulk cytoplasm to the vestibule of an individual hemichannel is $2 \times 10^{4}$ per second, suggesting that the zero wait state condition is a reasonable assumption.

\section{Abbreviations \\ CFP: Cyan Fluorescent Protein; CL: Cytoplasmatic loop; E1,E2: Extracelullar loop 1,2; hCx26wt: Wild type human connexin 26; hCx30wt: Wild type human connexin 30; ICS: Intra cellular solution; MD: Molecular dynamics; NT: N terminus; NTV-ensemble: Ensemble with fixed number of particles, volume and temperature; PMF: Potential of mean force; SEM: Standard error of the mean; TM1 to 4: Transmembrane helix 1 to 4.}

\section{Competing interests}

The authors declare that they have no competing interests.

\section{Authors' contributions}

FZ Designed and performed simulations. Analyzed results the of simulations and drafted the manuscript. GP Designed and performed simulations. MFS Performed part of the experiments and simulations. MB Coordinated the experimental work and analyzed the results. FM Coordinated the work. Analyzed results, drafted and revised the manuscript. All authors read and approved the final manuscript.

\section{Acknowledgements}

We thank Giuseppe Zanotti (Dept. of Biological Chemistry, University of Padua) for useful discussions.

Supported by MIUR PRIN grant no. 2009CCZSES and Telethon grant GGP09137 to FM, and from a University of Padua grant to FZ (prot. GRIC101108).

Computer simulations were performed at the CINECA and CASPUR supercomputer centers.

Pictures of the connexin at molecular level have been obtained by use of Visual Molecular Dynamics (VMD) software.

\section{Author details}

'Department of Physics and Astronomy "G. Galilei", University of Padua, Padua 35131, Italy. ${ }^{2}$ Venetian Institute of Molecular Medicine, Padua 35129, Italy. International school for advanced studies (SISSA), Trieste 34136, Italy. ${ }^{4}$ CNR Institute of Neurosciences, Padua Section, Padua 35131, Italy.

Received: 20 November 2012 Accepted: 12 February 2013 Published: 27 February 2013

\section{References}

1. Goodenough DA, Paul DL: Gap junctions. Cold Spring Harb Perspect Biol 2009, 1:a002576

2. Kar R, Batra N, Riquelme MA, Jiang JX: Biological role of connexin intercellular channels and hemichannels. Arch Biochem Biophys 2012, 524:2-15.

3. Harris AL: Connexin channel permeability to cytoplasmic molecules. Prog Biophys Mol Biol 2007, 94:120-143. doi:10.1016/j.pbiomolbio.2007.03.011.

4. Ek-Vitorin JF, Burt JM: Structural basis for the selective permeability of channels made of communicating junction proteins. Biochim Biophys Acta 2013, 1828:51-68.

5. Maeda S, Nakagawa S, Suga M, Yamashita E, Oshima A, Fujiyoshi Y, Tsukihara T: Structure of the connexin 26 gap junction channel at $3.5 \mathrm{~A}$ resolution. Nature 2009, 458:597-602.

6. Unger VM, Kumar NM, Gilula NB, Yeager M: Three-dimensional structure of a recombinant gap junction membrane channel. Science 1999, 283:1176-1180.

7. Fleishman SJ, Unger VM, Yeager M, Ben-Tal N: A Calpha model for the transmembrane alpha helices of gap junction intercellular channels. $\mathrm{Mol}$ Cell 2004, 15:879-888.

8. Kovacs JA, Baker KA, Altenberg GA, Abagyan R, Yeager M: Molecular modeling and mutagenesis of gap junction channels. Prog Biophys $\mathrm{Mol}$ Biol 2007, 94:15-28.

9. Pantano S, Zonta F, Mammano F: A fully atomistic model of the Cx32 connexon. PLoS One 2008, 3:e2614.

10. Kwon T, Harris AL, Rossi A, Bargiello TA: Molecular dynamics simulations of the Cx26 hemichannel: evaluation of structural models with Brownian dynamics. J Gen Physiol 2011, 138:475-493.

11. Zonta F, Polles G, Zanotti G, Mammano F: Permeation pathway of homomeric connexin 26 and connexin 30 channels investigated by molecular dynamics. J Biomol Struct Dyn 2012, 29:985-998.

12. Scott CA, Kelsell DP: Key functions for gap junctions in skin and hearing. Biochem J 2011, 438:245-254.

13. Hernandez VH, Bortolozzi M, Pertegato V, Beltramello M, Giarin M, Zaccolo M, Pantano S, Mammano F: Unitary permeability of gap junction channels to second messengers measured by FRET microscopy. Nat Methods 2007, 4:353-358.

14. Bastianello S, Ciubotaru CD, Beltramello M, Mammano F: Dissecting key components of the $\mathrm{Ca} 2+$ homeostasis game by multi-functional fluorescence imaging. Three-Dimensional and Multidimensional Microscopy: Image Acquisition and Processing XI, Proc. SPIE 5324 2004, 5:265-274. doi:10.1117/12.553731.

15. Chandler D: Statistical mechanics of isomerization dynamics in liquids and the transition state approximation. J Chem Phys 1978, 68:2959-2971.

16. Roux B, Karplus M: Ion-transport in a gramicidin-like channel - dynamics and mobility. J Phys Chem 1991, 95:4856-4868.

17. Kirkwood JG: Statistical mechanics of fluid mixtures. J Chem Phys 1935, 3:300-314.

18. Torrie GM, Valleau JP: Nonphysical sampling distributions in Monte Carlo free-energy estimation: Umbrella sampling. J Comput Phys 1977, 23:187-199.

19. Zwanzig R: Diffusion past an entropy barrier. J Phys Chem 1992, 96:3926-3930.

20. Locke D, Bian S, Li H, Harris AL: Post-translational modifications of connexin26 revealed by mass spectrometry. Biochem J 2009, 424:385-398.

21. Oshima A, Tani K, Hiroaki Y, Fujiyoshi Y, Sosinsky GE: Three-dimensional structure of a human connexin26 gap junction channel reveals a plug in the vestibule. Proc Natl Acad Sci USA 2007, 104:10034-10039.

22. Bukauskas FF, Jordan K, Bukauskiene A, Bennett MV, Lampe PD, Laird DW, Verselis VK: Clustering of connexin 43-enhanced green fluorescent protein gap junction channels and functional coupling in living cells. Proc Natl Acad Sci USA 2000, 97:2556-2561.

23. Palacios-Prado N, Sonntag S, Skeberdis VA, Willecke K, Bukauskas FF: Gating, permselectivity and $\mathrm{pH}$-dependent modulation of channels formed by connexin57, a major connexin of horizontal cells in the mouse retina. J Physiol 2009, 587:3251-3269.

24. Palacios-Prado N, Briggs SW, Skeberdis VA, Pranevicius M, Bennett MV, Bukauskas FF: pH-dependent modulation of voltage gating in connexin45 homotypic and connexin45/connexin43 heterotypic gap junctions. Proc Natl Acad Sci USA 2010, 107:9897-9902.

25. Schuttelkopf AW, van Aalten DM: PRODRG: a tool for high-throughput crystallography of protein-ligand complexes. Acta Crystallogr D Biol Crystallogr 2004, 60:1355-1363.

26. Case DA, Cheatham TE 3rd, Darden T, Gohlke H, Luo R, Merz KM Jr, Onufriev A, Simmerling C, Wang B, Woods RJ: The Amber biomolecular simulation programs. J Comput Chem 2005, 26:1668-1688. 
27. Roux B: The calculation of the potential of mean force using computersimulations. Comput Phys Commun 1995, 91:275-282.

28. Souaille M, Roux B: Extension to the weighted histogram analysis method: combining umbrella sampling with free energy calculations. Comput Phys Commun 2001, 135:40-57.

29. Hess B, Kutzner C, van der Spoel D, Lindahl E: GROMACS 4: Algorithms for highly efficient, load-balanced, and scalable molecular simulation. Journal of Chemical Theory and Computation 2008, 4:435-447.

30. Berendsen HJC, Postma JPM, van Gunsteren WF, Dinola A, Haak JR: Molecular dynamics with coupling to an external bath. J Chem Phys 1984, 81:3684-3690

31. Darden T, York D, Pedersen L: Particle mesh Ewald: An N $\log (\mathrm{N})$ method for Ewald sums in large systems. J Chem Phys 1993, 98:10089-10093.

32. Eyring $\mathrm{H}$ : The activated complex and the absolute rate of chemical reactions. Chem Rev 1935, 17:65-77.

33. Ermak DL, McCammon JA: Brownian dynamics with hydrodynamic interactions. J Chem Phys 1978, 69:1352-1361

34. Brown EB, Wu ES, Zipfel W, Webb WW: Measurement of molecular diffusion in solution by multiphoton fluorescence photobleaching recovery. Biophys J 1999, 77:2837-2849.

doi:10.1186/1478-811X-11-15

Cite this article as: Zonta et al.: The 3.5 angström X-ray structure of the human connexin26 gap junction channel is unlikely that of a fully open channel. Cell Communication and Signaling 2013 11:15.

\section{Submit your next manuscript to BioMed Central and take full advantage of:}

- Convenient online submission

- Thorough peer review

- No space constraints or color figure charges

- Immediate publication on acceptance

- Inclusion in PubMed, CAS, Scopus and Google Scholar

- Research which is freely available for redistribution 